\title{
PHIPS-HALO: the airborne Particle Habit Imaging and Polar Scattering probe - Part 1: Design and operation
}

\author{
Ahmed Abdelmonem et al. \\ Correspondence to: Ahmed Abdelmonem (ahmed.abdelmonem@kit.edu) and Martin Schnaiter (martin.schnaiter@kit.edu)
}

The copyright of individual parts of the supplement might differ from the CC-BY 3.0 licence. 


\section{S1 Sensitivity of the probe to turbulence in the detection volume for non-zero angle of attack and angle of sideslip}

S1.1 Airflow conditions at the probe inlet under flight conditions

In the CFD simulation, due to the rotational symmetry of the inlet part of the PHIPS-HALO probe, angle of attack (AoA) and angle of sideslip (AoS) can be regarded as complementary. During real flights both angles are measured separately by the aircraft avionics. The probe is mounted with an attitude of $2.8^{\circ}$ nose down to compensate the average aircraft attitude during normal flight. An additional $1.6^{\circ}$ nose inboard attitude with respect to the longitudinal axis of the aircraft is applied to compensate for the diverting flow field in horizontal direction around the main aircraft body. Both values have been determined using CFD airflow studies by the manufacturer of the canisters holding the probes.

Figure S1 shows both angles and true airspeed from a measurement flight of HALO on 1st October 2014 over Brazil. The angle of attack has been compensated for the pre-tilted attitude of the probe mount. For comparison with the CFD simulation, the angle between the actual flow vector at the probe inlet and the probe's central axis can be calculated using the relation $\cos (\Varangle$ flow, probe $)=\cos (\mathrm{AoA}) \cdot \cos (\mathrm{AoS})$.

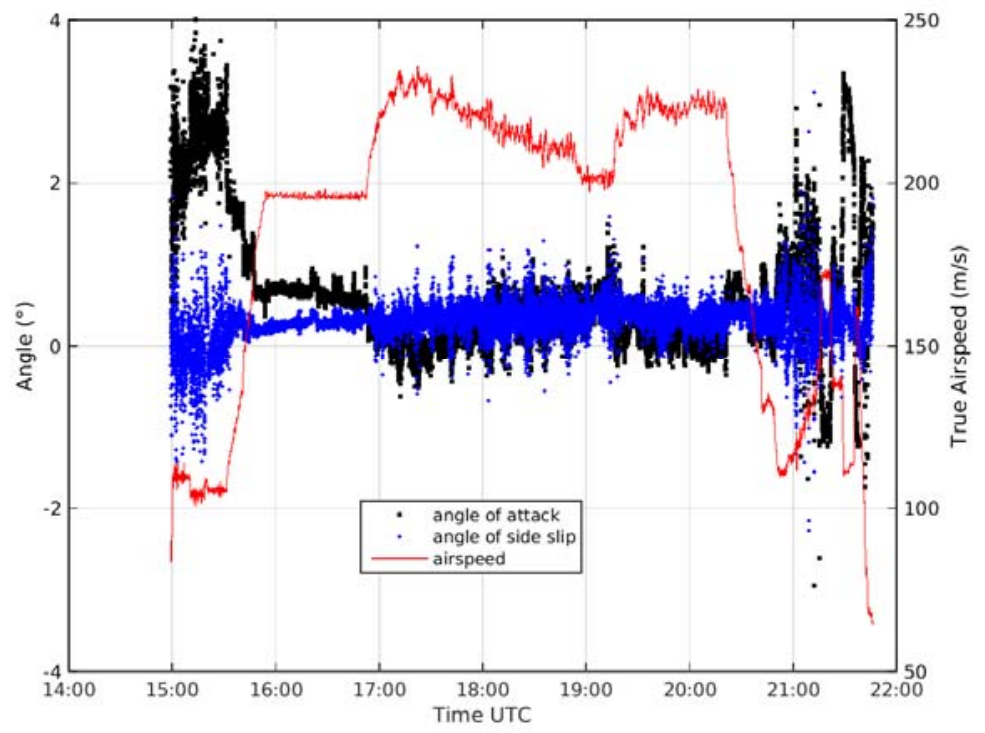

Figure S1 Airflow parameters at the HALO-PHIPS probe inlet inferred from avionics data of the HALO aircraft on a typical flight during the ACRIDICON campaign on 01.Oct.2014 over Brazil. The measured angle of attack has been corrected by subtracting $2.8^{\circ}$ of the pre-tilt probe attitude in vertical direction. 
S1.2 Probe sensitivity study to non-zero angle of attack

A series of simulations has been performed investigating the turbulent flow field inside the probe inlet as function of varying angle of attack or angle of sideslip. The simulation results shown here have been obtained applying typical flight conditions of the HALO aircraft (0.187 mbar ambient pressure, $227 \mathrm{~K}$ ambient temperature, $200 \mathrm{~m} \mathrm{~s}^{-1}$ flight speed).

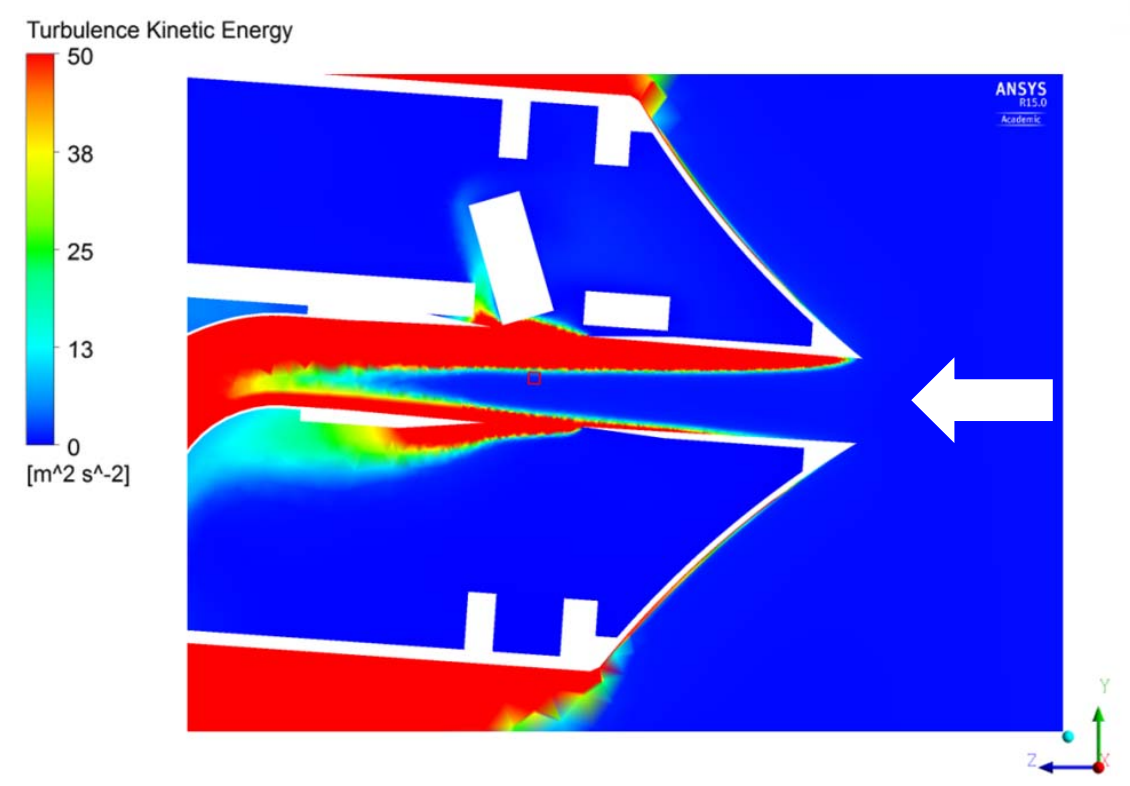

Figure S2 Turbulent kinetic energy in the probe inlet at a $4^{\circ}$ angle of attack. The red box represents the detection volume.

Figure S2 shows the turbulent kinetic energy in the probe inlet for a $4^{\circ}$ angle of attack. Due to the tilt of the probe with respect to the flow direction the turbulent region which originates from the upper inlet wall almost extends to the detection volume. 


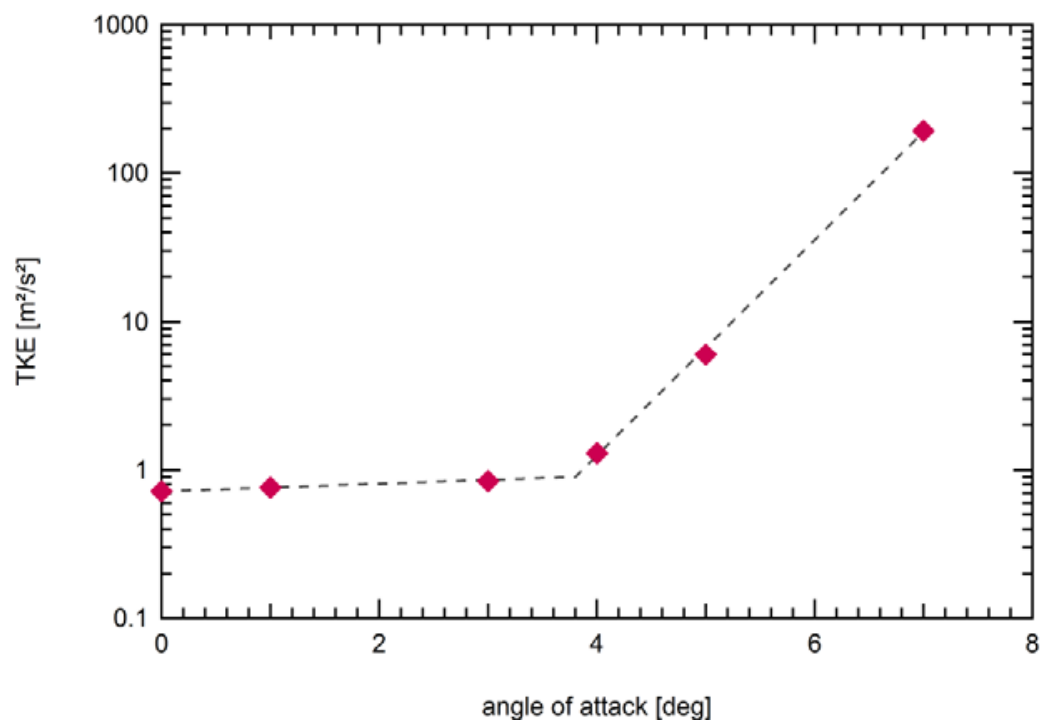

Figure S3 Turbulent kinetic energy at the detection volume as determined from CFD simulations from various angles of attack. Below $4^{\circ}$ angle of attack the TKE is at near background value but increases rapidly for larger angles.

Figure S3 shows the turbulent kinetic energy in the detection volume for various angles of attack. The graph indicates that the probe can be operated safely regarding turbulence in the detection volume for angles of attack within $\pm 4^{\circ}$. 